\title{
Galaxy Formation \& Dark Matter Modelling in the Era of the Square Kilometre Array
}

\author{
C. Power ${ }^{a \star}$, C.D.P. Lagos ${ }^{* \dagger \dagger}$, B. Qin ${ }^{c \ddagger}$, C.M. Baugh ${ }^{d}$, D. Cunnama ${ }^{e}$, J. Fu ${ }^{f}$, H.S. $\mathbf{K i m}^{g}$, \\ C.G. Lacey ${ }^{d}$, L. Li ${ }^{c}$, D. Obreschkow ${ }^{a}$, J. Wang ${ }^{c}$, Y. Wang ${ }^{c}$ and M. Zhu ${ }^{c}$
}

${ }^{a}$ International Centre for Radio Astronomy Research, The University of Western Australia, 35 Stirling Highway, Crawley, WA 6009, Australia

${ }^{b}$ European Southern Observatory,Karl-Schwarzschild-Str. 2, 85748 Garching bei München, Germany

${ }^{c}$ National Astronomical Observatories, Chinese Academy of Science, A20 Datun Road, Beijing 100012, China

${ }^{d}$ Institute for Computational Cosmology, Durham University, Science Laboratories, South Road, Durham DH1 3LE, United Kingdom

${ }^{e}$ Department of Physics, University of Western Cape, Bellville 7535, Republic of South Africa

${ }^{f}$ Shanghai Astronomical Observatory, Chinese Academy of Sciences, 80 Nandan Road, Shanghai 200030, China

${ }^{g}$ School of Physics, The University of Melbourne, Parville, Vic 3010, Australia

E-mail: * ${ }^{*}$ ris.power@icrar.org, ${ }^{\dagger}$ clagos@eso.org, ${ }^{\ddagger}$ inbo@bao.ac.cn

Theoretical galaxy formation models are an established and powerful tool for interpreting the astrophysical significance of observational data, particularly galaxy surveys. Such models have been utilised with great success by optical surveys such as 2dFGRS and SDSS, but their application to radio surveys of cold gas in galaxies has been limited. In this chapter we describe recent developments in the modelling of the cold gas properties in the models, and how these developments are essential if they are to be applied to cold gas surveys of the kind that will be carried out with the SKA. By linking explicitly a galaxy's star formation rate to the abundance of molecular hydrogen in the galaxy rather than cold gas abundance, as was assumed previously, the latest models reproduce naturally many of the global atomic and molecular hydrogen properties of observed galaxies. We review some of the key results of the latest models and highlight areas where further developments are necessary. We discuss also how model predictions can be most accurately compared with observational data, what challenges we expect when creating synthetic galaxy surveys in the SKA era, and how the SKA can be used to test models of dark matter.

Advancing Astrophysics with the Square Kilometre Array

June 8-13, 2014

Giardini Naxos, Italy

\footnotetext{
* Speaker.
} 


\section{Introduction}

Neutral hydrogen, both in its atomic $(\mathrm{HI})$ and molecular $\left(\mathrm{H}_{2}\right)$ forms, plays a fundamental role in galaxy formation, principally as the raw material from which stars are made. At any given time, the fraction of a galaxy's mass that is in the form of neutral hydrogen will be determined by the competing rates at which it is depleted (by e.g. star formation, photo-ionization, expulsion via winds) and replenished (by e.g. recombination, accretion from galaxy's surroundings) - physical processes that are the foundations of any viable theory of galaxy formation.

Most of what we know currently about $\mathrm{HI}$ in galaxies derives from 21-cm surveys of the nearby Universe $(z \lesssim 0.05)$ such as HIPASS (HI Parkes All Sky Survey; cf. Barnes et al., 2001) and ALFALFA (Arecibo Legacy Fast ALFA; cf. Giovanelli et al., 2005). Efforts are ongoing to complement these data with results from higher redshifts, using innovative techniques to estimate statistically typical galaxy HI content (e.g. stacking, cf. Lah et al. 2007 and Delhaize et al. 2013). These efforts will accelerate dramatically over the coming decade, with the advent of next generation radio telescopes such as the Australian Square Kilometre Array Pathfinder (ASKAP Johnston et al., 2008), MeerKAT (de Blok et al., 2009), and ultimately the Square Kilometre Array (SKA) itself. The SKA and its shorter wavelength counterpart the Atacama Large Millimetre Array (hereafter ALMA) offer the technical capability to chart the evolution of neutral hydrogen in galaxies over the last $\sim 12$ billion years of cosmic time. This is a significant fraction of the assembly history of present-day galaxies, and the results of these observations will provide a stringent test of our theories of galaxy formation and evolution.

These theories of galaxy formation and evolution have undergone some timely and fundamental changes over the last $\sim 5$ years. Signficantly for forthcoming $\mathrm{HI}$ and $\mathrm{H}_{2}$ galaxy surveys, there has been particular emphasis on model predictions for the cold gas content of galaxies, which has led to some important conceptual changes in, for example, the treatment of star formation in the models. In the remainder of this chapter, we review briefly the essential features of the theoretical galaxy formation models, in particular the semi-analytical approach (\$2); discuss the recent developments in these models, explaining why they were necessary (\$3) and what the consequences are ( $\$$ to $\S 7)$; and indicate where further developments will be necessary ( 88$)$. Finally, we explore the potential of the SKA as a testbed for our theories of dark matter (\$9).

\section{Galaxy Formation Modelling: Hydrodynamical and Semi-Analytical Approaches}

The two most widely used techniques to study galaxy formation in a cosmological context are hydrodynamical $N$-body simulations and semi-analytical models. Both follow the formation and evolution of dark matter halos over cosmic time using cosmological $N$-body simulations. However, hydrodynamical models compute the formation and evolution of the baryonic structures (e.g. hot gaseous halos, galaxy discs) at the same time as the dark matter, whereas semi-analytical models use the results of the pre-computed $N$-body simulation to guide the formation and evolution of the baryonic structures subsequently.

The methods should be viewed as complementary, each with their own advantages and disadvantages. Briefly, these are as follows. 


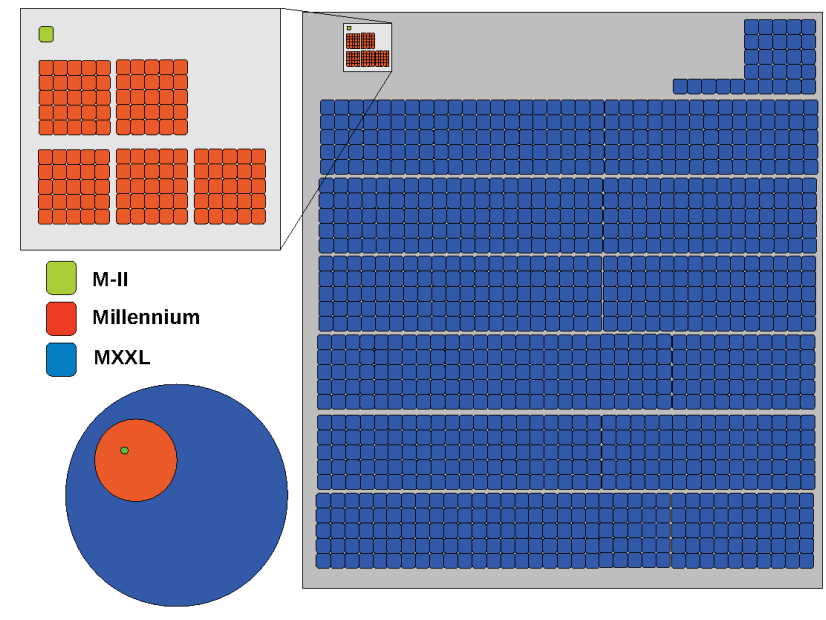

Figure 1: Schematic figure showing the difference in volume between the Millennium-I, II and XXL simulations (courtesy of Raul Angulo). The volume of Millennium-I exceeds that of Millennium-II by a factor of 125 times, while it is dwarfed by XXL by more than a factor of 200 in volume. The particle mass of Millennium-II is $\approx 1225$ times smaller than the XXL simulation. Galaxy formation models for the SKA will require Millennium-II resolution in Millennium-I or XXL volumes.

- Hydrodynamical $N$-body simulations capture the complexity of gas and stellar dynamics in a way that semi-analytical models cannot, which is invaluable when studying, for example, the details of gas accretion onto galaxies. They are, however, computationally expensive when compared to semi-analytical models, and usually employed when studying galaxy formation on the scale of individual systems or in small-to-intermediate cosmological volumes.

- Semi-analytical models are computationally inexpensive when compared to hydrodynamical simulations, requiring only the $N$-body simulation and derived merger histories for the host dark matter halos of galaxies. While they sacrifice capturing the complex dynamical and structural properties of galaxies, they are ideally suited to the study of statistical samples of galaxies in cosmological volumes over many cosmic epochs. The models are such that they can be parameterised and calibrated to reproduce a set of standard observations (e.g. the galaxy luminosity function, and the Tully-Fisher relation) in a relatively straightforward manner (see Baugh 2006 for a comprehensive review), a process that is comparatively unwieldy for hydrodynamical simulations.

Therefore, it is in the detailed dynamical and structural properties of baryons within galaxies and in the surrounding haloes that the methods differ ${ }^{1}$; otherwise both approaches treat the physics of galaxy formation - star formation, stellar winds and supernovae, black hole growth and outburst of feedback - using similar simplified prescriptions that parameterise physical processes on spatial scales much smaller than can be resolved in the simulations.

This distinction is important when considering the kinds of volumes $\left(1 \mathrm{Gpc}^{3}\right.$ and larger) that the SKA will be probing. To date, the state-of-the-art in cosmological hydro-dynamical galaxy

\footnotetext{
${ }^{1}$ Although we note that efforts are underway in the semi-analytical models to capture, for example, the effect of baryon displacement highlighted by e.g. van Daalen et al. (2014) in hydrodynamical simulations
} 
formation simulations probe volumes of order $100 \mathrm{Mpc}^{3}$ (Vogelsberger et al. 2014; Schaye et al. 2014). Contrast with semi-analytical models, which have been applied to the Millennium series of simulations. These are some of the largest cosmological $N$-body (i.e. dark matter only) simulations ever run, both in terms of particle number and volume - spanning the Millennium-I Simulation (Springel et al. 2005; with particle mass of $1.2 \times 10^{9} h^{-1} \mathrm{M}_{\odot}$ and box size of $500 h^{-1} \mathrm{Mpc}$ ), the Millennium-II Simulation (Boylan-Kolchin et al. 2009; with particle mass of $6.9 \times 10^{6} h^{-1} \mathrm{M}_{\odot}$ and box size of $100 h^{-1} \mathrm{Mpc}$ ) and Millennium XXL (Angulo et al. 2012; with particle mass of $8.5 \times 10^{9} h^{-1} \mathrm{M}_{\odot}$ and box size of $3000 h^{-1} \mathrm{Mpc}$ ). Fig. 1 shows these three simulations in a way that emphasises the differences in volumes modelled.

Fig. 1 also encapsulates the challenge facing galaxy formation modelling in the SKA era. The SKA will probe enormous volumes, in excess of those modelled in the Millennium-I and comparable to those in Millennium XXL, but most HI in the Universe is concentrated in low-mass galaxies and dark matter halos; this requires high mass resolution, comparable to that of the Millennium-II. This combination of high mass resolution and enormous volume demands a semi-analytical rather than a hydrodynamical approach. Hydrodynamical simulations will play a vital role in informing semi-analytic models, both about the limitations of assumptions as well as improvements and approximations that can be made (some aspects of which we discuss in Section 8). However, it is semi-analytical models that we expect to underpin the theoretical framework supporting the SKA and it is this approach that we focus on in the remainder of this chapter.

\section{Semi-Analytical Modelling of HI in Galaxies}

As noted already, semi-analytical models offer a simplified physically motivated treatment of the processes that control the amount of cold gas ${ }^{2}$ in a galaxy: namely the rate at which it cools radiatively within its host dark matter halo; the rate at which it is accreted in galaxy mergers; the rate at which it is consumed in star formation; and the rate at which it is reheated or expelled from galaxies by sources of feedback (e.g. photo-ionisation, stellar winds, supernovae and active galactic nuclei, AGN, heating).

A visual impression of the results of a semi-analytical calculation is given in Fig. 2, which shows the stellar and $\mathrm{HI}$ content of galaxies in a cluster drawn from the Millennium-I Simulation, as predicted by the Lagos et al. (2011a) model. The Lagos et al. (2011a) model was one of the first to predict the $\mathrm{HI}$ and $\mathrm{H}_{2}$ content of galaxies explicitly and self-consistently, tracking individual gas phases over the duration of the calculation; prior to this, $\mathrm{HI}$ and $\mathrm{H}_{2}$ masses in galaxies were obtained by post-processing cold gas masses at the end of the calculation. We consider briefly the results of these older post-processed models, and the physics underpinning the newer self-consistent models.

Post-processed models predicted galaxy populations with cold gas properties in broad agreement with observations. Baugh et al. (2004) and Rawlings et al. (2004) showed that semi-analytical mass functions of HI in galaxies (hereafter HIMF) were in satisfactory agreement with observations (HIPASS; cf. Zwaan et al., 2003) and semi-empirical fits. They converted between cold gas mass and $\mathrm{HI}$ mass by using the observationally measured global densities of $\mathrm{HI}$ and $\mathrm{H}_{2}$ (Zwaan et al. 2003 and Keres et al. 2003 respectively) and splitting the cold gas mass into $\mathrm{HI}, \mathrm{H}_{2}$ and He phases.

\footnotetext{
${ }^{2}$ Here cold gas refers to gas that has cooled radiatively from a hot phase to below $10^{4} \mathrm{~K}$, is predominately $\mathrm{HI}, \mathrm{H}_{2}$ and helium (He), and is available for star formation.
} 


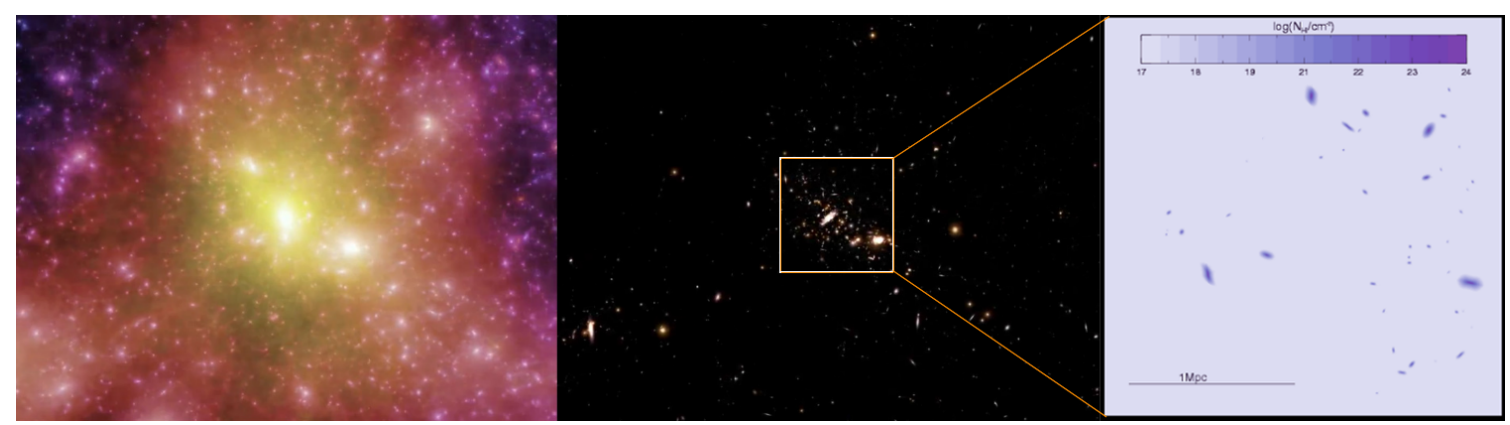

Figure 2: A massive galaxy cluster drawn from the Millennium-I Simulation, showing colour coded projected dark matter density, galaxy optical light and HI content (left, middle and right panels respectively). Galaxy properties were calculated using the GALFORM semi-analytical model of Lagos et al. (2011a). Image of dark matter distribution courtesy of Volker Springel.
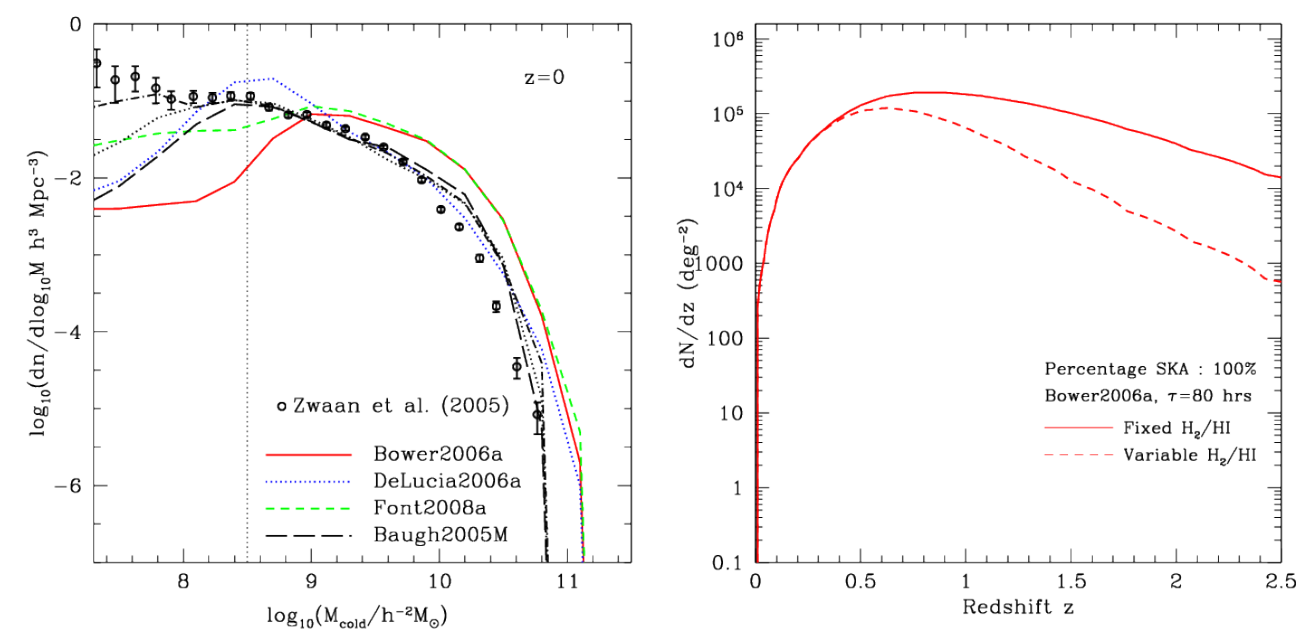

Figure 3: Left Panel: Cold gas mass function at $z=0$ in 4 different galaxy formation models applied to the Millennium-I Simulation (see legend); data points are observational estimates derived from HIPASS (Zwaan et al., 2005) obtained by rescaling HI masses by a factor of $\sim 1.85$; see text for further details. Dotted vertical line corresponds to the resolution limit of the models. Right Panel: Predicted number counts of galaxies per square degree per unit redshift for peak flux limited 1-year survey on a fiducial SKA with effective area 1 $\mathrm{km}^{2}$ and integration time of $\tau=80 \mathrm{hrs}$, based on Bower et al. (2006). Solid (dashed) curves correspond to a fixed (variable) conversion factor from cold gas to $\mathrm{H}_{2} / \mathrm{HI}$. Both figures from Power et al. (2010).

Obreschkow \& Rawlings (2009) and Obreschkow et al. (2009) adopted a more sophisticated approach to conversion, using the empirical relation between the $\mathrm{HI} / \mathrm{H}_{2}$ ratio and midplane pressure in galaxies (cf. Blitz \& Rosolowsky, 2006) to predict the $\mathrm{HI}, \mathrm{H}_{2}$ and He phases on a galaxy-bygalaxy basis. Power et al. (2010) compared results from four then favoured models (Baugh et al., 2005; Bower et al., 2006; De Lucia \& Blaizot, 2007; Font et al., 2008) applied to the Millennium-I Simulation for the mass function (MF) of cold neutral gas (atomic and molecular) in galaxies as a function of redshift. Despite different implementations of galaxy formation physics, the predictions were found to be broadly consistent with one another; key differences reflected how the models treated AGN feedback and the timescale for star formation as a function of redshift. The 


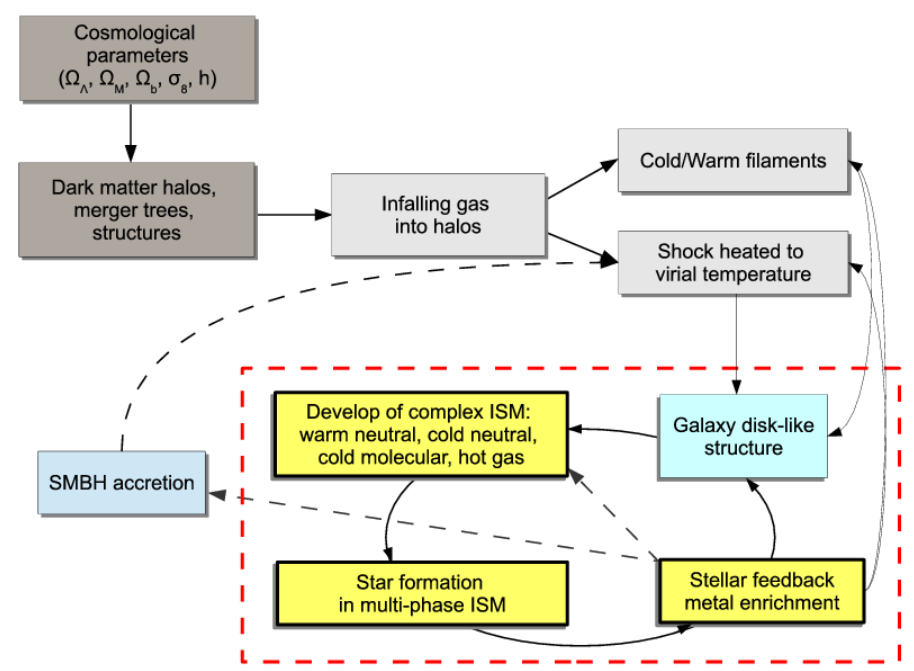

Figure 4: Schematic of the Physics of Disc Galaxy Formation and the ISM. Galaxies form within the potential wells of dark matter halos, whose formation is governed by the cosmological parameters and dark matter model, from baryons; these baryons either shock-heated upon infall and cooled or fell directed onto the galaxy along filaments. This emphasises the impact modelling of the ISM and SF has on galaxy components and the circum- and inter-galactic medium.

predicted MF of cold gas in galaxies agreed well with that inferred from HI surveys at $z=0$ (see left panel of Fig. 3$)^{3}$.

However, consideration of how the HIMF evolves with cosmic time and what this implies for future $\mathrm{HI}$ surveys highlighted that uncertain conversions from cold gas mass to $\mathrm{HI}$ and $\mathrm{H}_{2}$ masses translate into large variations in model predictions. The right panel of Fig. 3 shows how predicted HI number counts are influenced by the choice of conversion factor - the solid (dashed) curve assumes Baugh et al. (2004) (Obreschkow et al. 2009) approaches. Number counts differ negligibly for $z \lesssim 1$, but grow to a factor of $\sim 10$ by $z \sim 2.5$, reflecting the increasing proportion of cold gas in the form of $\mathrm{H}_{2}$ with increasing $z$, as galaxies become more gas-rich and more compact.

Self-consistent models have addressed the question of how to convert from a cold gas mass to $\mathrm{HI}$ and $\mathrm{H}_{2}$ mass by focusing on improved modelling of the interstellar medium (ISM), and, in particular, the relationship between star formation (SF) and neutral hydrogen phases; this has been the pivotal area of development in semi-analytical models over the last $\sim 5$ years. Post-processed models treated the ISM as a single star-forming phase (e.g. Cole et al. 2000), but this is at odds with both empirical evidence and theoretical expectation. Observationally, there is evidence that a galaxy's SF rate (SFR) per unit area and molecular gas surface density correlate linearly, $\Sigma_{\mathrm{SFR}} \propto$ $\Sigma_{\text {mol }}$ (Wong \& Blitz 2002; Bigiel et al. 2008). Theoretically, recent hydrodynamical simulations of star forming clouds suggests that the physical conditions necessary for SF - high density and low temperature gas - are also necessary for formation of $\mathrm{H}_{2}$ and $\mathrm{CO}$, but these conditions are driven by the presence of dust shielding, which prevents photo-dissociation by energetic UV photons, and

\footnotetext{
${ }^{3}$ Here the HIPASS HIMF of Zwaan et al. 2005 was converted to a cold gas MF by an empirically-motivated rescaling of $\mathrm{HI}$ masses by a factor of $\sim 1.85$. This accounts for the $24 \%$ helium and assumes that $\mathrm{HI}$ and $\mathrm{H}_{2}$ are split such that the global $\mathrm{HI}$ and $\mathrm{H}_{2}$ densities are consistent with the observational estimates of Zwaan et al. (2003) and Keres et al. (2003).
} 
CII cooling (Glover \& Clark, 2012a,b). In other words, the presence of $\mathrm{H}_{2}$ correlates naturally with SF (see also the arguments of Schaye 2004 and recent numerical work by Gnedin \& Kravtsov 2011; Feldmann et al. 2011; Glover \& Clark 2012b; Krumholz 2013).

This has led to the development of semi-analytical models that implement a fully self-consistent treatment of the ISM and SF (cf. Cook et al. 2010; Fu et al. 2010; Lagos et al. 2011b; also Robertson \& Kravtsov 2008; Dutton et al. 2010). Fig. 4 encapsulates the complex interplay of physical processes in the ISM and their influence on galaxy formation, ranging from star formation in high density regions (e.g. Bigiel et al. 2008), to supernovae-driven turbulence (e.g. Dobbs et al. 2011), to chemical enrichment of the inter-galactic medium via outflows (e.g. Putman et al. 2012), and it provides a template for the treatment of the ISM in the models.

Two of the first models to implement this self-consistent approach - Lagos et al. (2011b) and Fu et al. (2010) - each explored two approaches to estimating the $\mathrm{HI}$ and $\mathrm{H}_{2}$ content of the ISM ${ }^{4}$. The first was the empirical relation of Blitz \& Rosolowsky (2006), which relates the molecularto-atomic surface density ratio to hydrostatic pressure within the disc, estimating the SFR from the molecular gas surface density using the well measured molecular depletion timescale Bigiel et al. (2008) (also used by Cook et al. 2010). The second was the theoretical relation of Krumholz et al. (2009), which models SF as taking place in turbulent, marginally stable clouds, estimating the molecular abundance from the balance between the dissociating radiation flux and the formation of molecules on the surface of dust grains. Both Lagos et al. (2011b) and Fu et al. (2010) favoured the empirical relation of Blitz \& Rosolowsky (2006), which provides a better fit to the HIMF at $z=0$ (Zwaan et al. 2005; Martin et al. 2010) and predicts a clustering of HI selected galaxies in good agreement with observations (cf. Kim et al. 2013b); in contrast, the theoretical model of Krumholz et al. (2009) overpredicts the number density of intermediate HI mass galaxies.

In the remainder of this chapter, we assume self-consistent models that derive from Lagos et al. (2011b) and later. Model parameters are calibrated to reproduce the $z=0 \mathrm{~K}$ - and $\mathrm{b}_{j}$ band luminosity functions (hereafter LFs), which govern AGN and stellar feedback, and UV LFs at $z=2-6$, which limit the duration of starbursts; no gas properties were used.

\section{Insights into Global Star Formation Rates over Cosmic Time}

The more realistic treatment of the ISM in the newer self-consistent models leads naturally to predictions that can explain trends in observational data.

First, the observed steep decline of SFR density with decreasing redshift is a consequence of the steep decline in the underlying molecular gas mass density with redshift (see left and middle panels of Fig. 5; see also Lagos et al. 2011a); in contrast, HI mass density evolves very weakly with redshift (right panel of Fig. 5). This arises from a combination of decreasing gas fractions and increasing galaxy sizes with decreasing redshift, which both act to reduce the gas surface density and the hydrostatic pressure of the disc. The redshift evolution of SFR density tracks that of the gas surface density of galaxies dominating the SFR in the Universe at a given epoch.

Increasing hydrostatic pressure in galaxy discs and increasing gas fractions with increasing redshift also leads to increasing molecular to dynamical mass ratios, which is consistent with ob-

\footnotetext{
${ }^{4}$ The Lagos et al. (2011b) and Fu et al. (2010) follow similar prescriptions, the key difference being that Fu et al. (2010) follow the radial gas profile of the galaxy disc explicitly.
} 

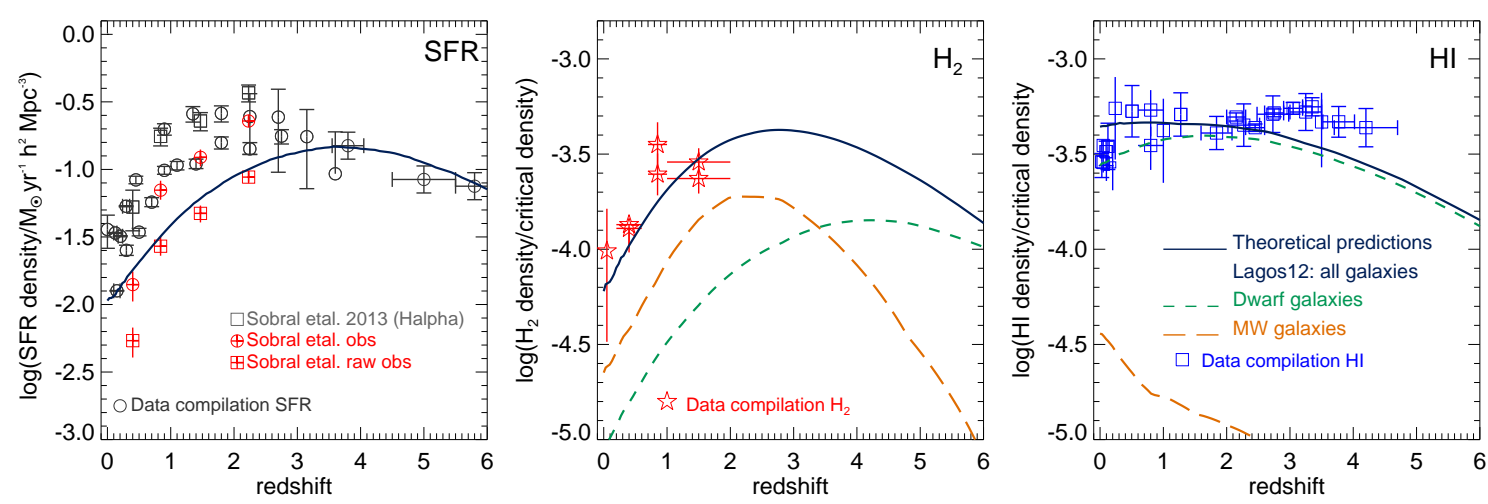

Figure 5: Global densities (solid lines) of the SFR in units of $M_{\odot} \mathrm{yr}^{-1} h^{3} \mathrm{Mpc}^{-3}$ (left hand panel), the estimated molecular hydrogen (middle panel) and the atomic hydrogen (right hand panel) in units of the critical density, as a function of redshift for the Lagos et al. (2012) model. The circles, stars and squares correspond to a data compilation of observations presented in Lagos et al. (2014) for the SFR, $\mathrm{H}_{2}$ and HI densities, and are taken from Sobral et al. (2013). We also show the predicted contribution from dwarf galaxies (those with stellar masses $<10^{9} M_{\odot}$; dashed lines) and Milky-Way (MW) type galaxies (those with stellar masses in the range $10^{10} M_{\odot}-10^{11} M_{\odot}$; long-dashed lines).

servations of normal galaxies in the Universe up to $z \sim 2.5$ (see Geach et al. 2011). Note, however, that the increase in the SFR density is $2-3$ times higher than that of the $\mathrm{H}_{2}$ density; this reflects the greater efficiency of star formation in starbursts, which is not accounted for in the normal star-forming discs assumed in the model (Lagos et al. 2014). Lagos et al. (2011a) showed that the shape of the relation between molecular gas fraction (i.e. $M_{\mathrm{mol}} / M_{\mathrm{mol}}+M_{*}$ ) and redshift depends on galaxy environment, such that, on average, galaxies with low mass host halos have larger molecular gas fractions than those with more massive hosts. Gas dynamical simulations agree qualitatively with this prediction (e.g. Davé et al. 2011).

The offset between the observational data points and model prediction evident in the left panel of Fig. 5 is well known in galaxy formation models, which tend to under-predict the SFR density by factors of $\sim 2-3$. This discrepancy has been explored in some depth in Lagos et al. (2014) and is driven by a number of factors, including extrapolation of observed luminosity functions to account for the contribution of faint, undetected galaxies; the adopted stellar initial MF; and uncertainties in the SFRs of individual galaxies, which are of order 0.3-0.4 dex, as found by Speagle et al. (2014).

Second, the molecular-to-atomic gas ratio correlates with stellar mass, and so the low-mass end of the HIMF is steeper than the low-mass end of the stellar and $\mathrm{H}_{2}$ MFs. We show in Fig. 5 the predicted contribution to the $\mathrm{HI}$ and $\mathrm{H}_{2}$ densities from low mass galaxies (dwarfs) and more massive, Milky-Way like galaxies; the contribution from Milky-Way like galaxies to HI density is negligible except at very low redshifts, while dwarf galaxies dominate over the entire redshift range. For $\mathrm{H}_{2}$, we see that Milky Way like galaxies dominate at lower redshifts but dwarfs make a significant contribution at higher redshifts, when the contribution from Milky Way like galaxies declines sharply. Note that these estimates are derived from the observations of Keres et al. (2003) and Berta et al. (2013) and required a number of assumptions to be made in their estimation (e.g. correlation between $A_{\mathrm{UV}}$ and $\mathrm{H}_{2}$ ). Fig. 5 suggests that the SKA will provide new and interesting insights into dwarf galaxies, which at present are only partially accessible optical and near infrared 


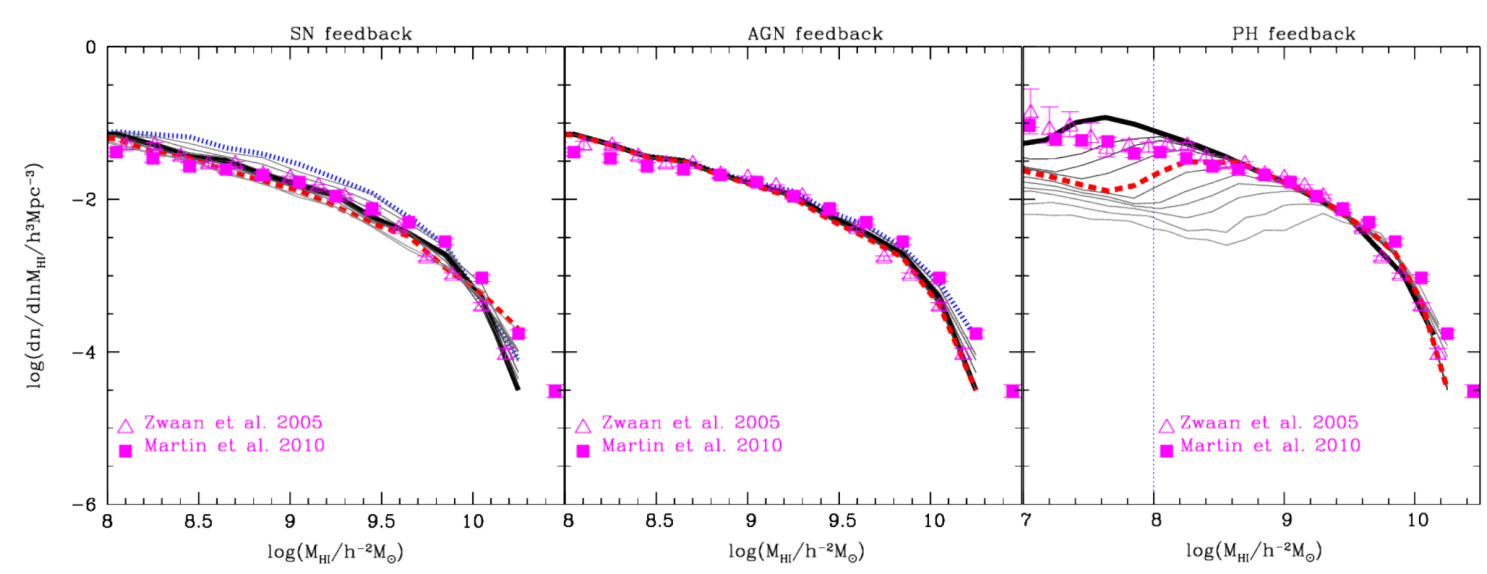

Figure 6: Impact of feedback from supernovae (SNe), AGN and the photoionising background (PH; left to right panels) on the HIMF in the Lagos et al. (2011a) model. Open triangles (filled squares) represent observational data from the HIPASS Zwaan et al. (2005)) and ALFALFA Martin et al. (2010) surveys. SNe strength varies in the range of $V_{\text {hot }}=300 \mathrm{~km} / \mathrm{s}$ to $V_{\text {hot }}=700 \mathrm{~km} / \mathrm{s}$; AGN strength varies in the range of $\alpha_{\text {cool }}=0.4-0.8$; photoionisation strength in the range of $30 \mathrm{~km} / \mathrm{s} \leq V_{\text {cut }} \leq 90 \mathrm{~km} / \mathrm{s}$. Dark solid lines correspond to the default models values; red dashed and blue dotted curves correspond strong and weak feedback limits, respectively.

telescopes.

\section{Probing the Physics of Feedback with HI Surveys}

Semi-analytical models include a range of physical processes that either prevent hot gas from cooling onto a galaxy or heat up neutral gas and expel it from a galaxy (cf. Baugh, 2006). Simple physical arguments to detailed numerical calculations demonstrate that supernovae are responsible for expelling large amounts of gas from galaxies in low mass halos $\left(M_{\text {halo }}<\right.$ few $\left.\times 10^{11} M_{\odot}\right)$, shaping the faint-end of the LF. Similarly, the photo-ionising background present during cosmological reionization quenches low-mass galaxy formation, influencing the faint-end of the LF (e.g Benson et al., 2002). AGN appear necessary to suppress excessive star formation in high stellar mass galaxies and so regulate the amplitude and shape of the bright-end of the LF (cf. Bower et al., 2006; Croton et al., 2006).

The effect of different feedback mechanisms on optical and near infrared LFs have been explored with semi-analytical models Benson et al. (2003); Baugh et al. (2005); Bower et al. (2006); Croton et al. (2006), and this has been extended to the HIMF by Kim et al. (2013b) using the Lagos et al. (2011a) model. These authors studied the influence of varying the strength of supernovae (SNe), AGN and photo-ionizing (PH) feedback on both the (optical) $b_{j}$-band LF and the HIMF; we focus on the HIMF in Fig. 6. As the Figure reveals, SNe feedback (left panel) affects the HIMF uniformly across the entire range of HI masses; weaker feedback boosts the amplitude of the HIMF, and vice versa. AGN (middle panel) have little impact on the HIMF except at the very highest HI masses, with weaker feedback reducing the slope. Interestingly, photo-ionization ( $\mathrm{PH}$; right panel) has a significant effect at lower HI masses, driven by the sensitivity of the low-mass end slope to 
the halo mass scale below which galaxy formation is suppressed at reionization. Here the caveat is that particular models for feedback were assumed.

\section{Clustering of HI-Selected Galaxies}

Both the older post-processed models and the newer self-consistent ones predict clustering of $\mathrm{HI}-$ selected galaxies that are broadly consistent with observations, which indicate that the clustering strength of HI-selected galaxies is weaker than that of their optically-selected counterparts (cf. Meyer et al., 2007; Martin et al., 2012). For example, Kim et al. (2011) and Kim et al. (2013b) demonstrated that the post-processed Bower et al. (2006) and Font et al. (2008) models ${ }^{5}$ and the self-consistent Lagos et al. (2011a) model, all variants of the GALFORM model of Cole et al. (2000), make predictions that are in good agreement with the observed 2-point correlation functions (cf. left panel of Fig. 7, with data points drawn from Meyer et al. 2007). The models suggest that it is the central galaxies of halos with masses $\approx 10^{11} h^{-1} \mathrm{M}_{\odot}$, which are more likely to be HI-rich, that determine the shape of the 2-point correlation function.

Interestingly, the Font et al. (2008) model, which seeks to capture the effects of ram-pressure stripping on the ISM of satellites and the consequences of this for their observed colours, predicts stronger clustering on small scales than the Bower et al. (2006) and Lagos et al. (2011a) models, which provide a better description of the observed data points. This suggests that treating the ISM of a galaxy either explicitly in its $\mathrm{HI}$ and $\mathrm{H}_{2}$ phases or simply as a monolithic cold gas phase has little effect on predicted clustering. Physically we expect how the ISM is modelled to influence how it is stripped - lower column density HI should be stripped preferentially while higher column density $\mathrm{H}_{2}$ should resist stripping, which is what is found in hydrodynamical simulations (e.g. Quilis et al., 2000; Bekki, 2014) - although it is plausible that the behaviour of a single phase cold gas ISM and a multiphase ISM in which $\mathrm{H}_{2}$ is retained is similar. Nevertheless, the treatment of the ISM in even the newer models is too coarse-grained to capture this, and ram pressure stripping modelling requires further development (e.g. Book \& Benson, 2010).

The models also predict that the halo occupation distribution (HOD) for HI galaxies (the mean number of galaxies with a given HI mass per host dark matter halo as a function of host halo mass; cf. right hand panel of Fig. 7, based on the Lagos et al. 2011a model) has a functional form that is distinct from that usually employed for optically selected galaxies (e.g. Wyithe \& Brown, 2010). We show Fig. 7 the HOD for HI-selected galaxies (heavy solid curve) split into centrals (heavy long-dashed curve) and satellites (heavy short-dashed curve); for comparison we show also the HOD for optically-selected ( $r$-band) galaxies (light solid curve), with light long- and short-dashed curves indicating centrals and satellites respectively. The contribution to the HI-selected HOD from centrals is negligible at higher halo masses $\left(\gtrsim 10^{13} h^{-1} \mathrm{M}_{\odot}\right)$, whereas the measured HOD in the $r$-band is consistent with the usual functional form assumed in the literature. Reconstructing the HOD of HI-selected galaxies will provide an important test of the treatment of ram pressure stripping of the models.

\footnotetext{
${ }^{5}$ Their cold gas masses were converted to HI masses using the approach of Baugh et al. (2004), which is tuned to recover the global densities of $\mathrm{HI}$ and $\mathrm{H}_{2}$ at $z=0$.
} 

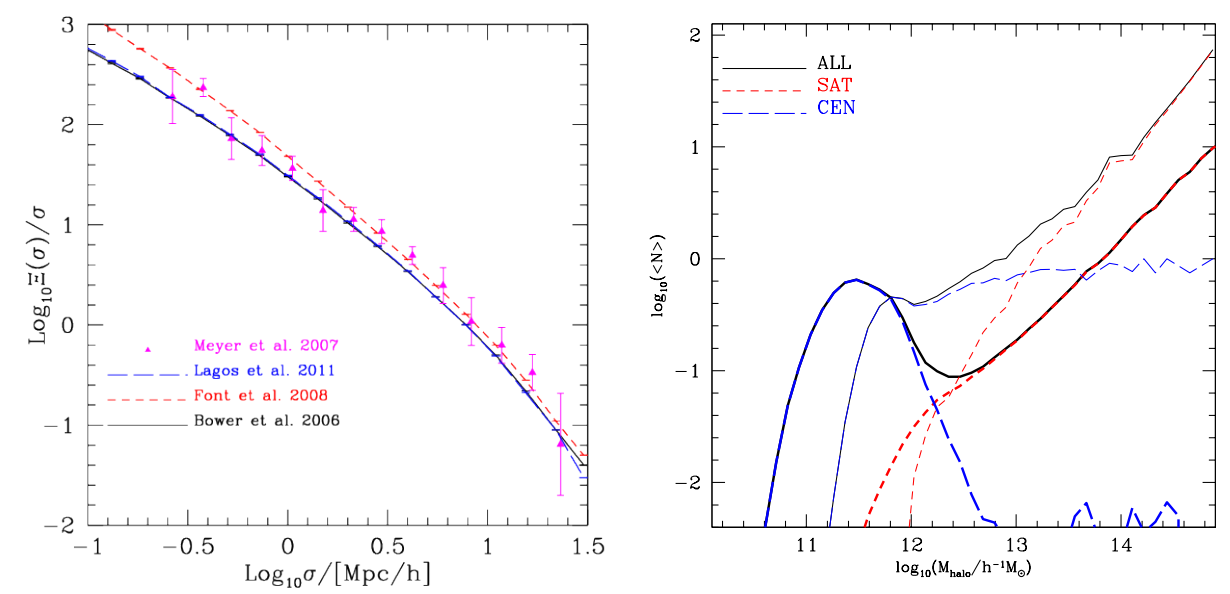

Figure 7: Predicted spatial clustering of HI galaxies. The left panel shows the projected 2-point correlation function of HI-rich galaxies at $z=0$, with symbols and errorbars indicating observational data from Meyer et al. (2007). Lines show predictions from three different models, as labelled, for galaxies with HI masses $M_{\mathrm{HI}}>10^{9.25} h^{-2} M_{\odot}$, which is consistent with threshold assumed in the observational measurement. The right panel shows the halo occupation distribution for HI-rich galaxies (heavy curves) and $r$-band selected galaxies (light curves) in the Lagos et al. (2011a) model, using the same data as in the left panel. ALL, SAT and CEN refer to all, satellite and central galaxies respectively.

\section{Probing Galaxy Kinematics with HI}

The baryonic content of galaxies in the local Universe can be probed using the Tully-Fisher relation between galaxy velocity width measured by $\mathrm{HI}$ in emission and baryonic mass (see left panel of Fig. 8). The dramatic increase in the number of galaxies with good quality HI emission lines measured by the SKA will allow us to test our understanding of galaxy kinematics for statistical samples of galaxies as a function of epoch with the most stringent data ever available. Semianalytical models have proven to be particularly useful in modelling HI-derived kinematics, with work presented in Obreschkow et al. (2009), Obreschkow \& Rawlings (2009) and most recently Obreschkow et al. (2013) making it possible to build catalogues including complete HI emission lines (with velocity width, peak flux, and integrated flux) for millions of galaxies in a semi-analytic model derived from the Millennium-I Simulation (cf. Obreschkow et al., 2009).

Using this catalogue, the consistency of the galaxy velocity function (i.e. comoving number density of galaxies with a given rotational velocity) predicted in a Cold Dark Matter (CDM) universe with observations has been assessed.Obreschkow et al. (2013) replicated selection effects and completeness of observed galaxies to show that the reported discrepancy between the predicted and observed velocity functions claimed in the literature (e.g. Zwaan et al., 2010) is more apparent than real, arising from observational incompleteness and erroneous corrections to the observed data to recover galaxy velocities. The right panel of Fig. 8 shows the distribution of HI velocity widths predicted by the model of Obreschkow et al. (2009) after taking into account sky coverage, completeness and blending of sources, compared to observations. The excellent agreement suggests that comparison of predicted and observed velocity functions must be to directly model HI 

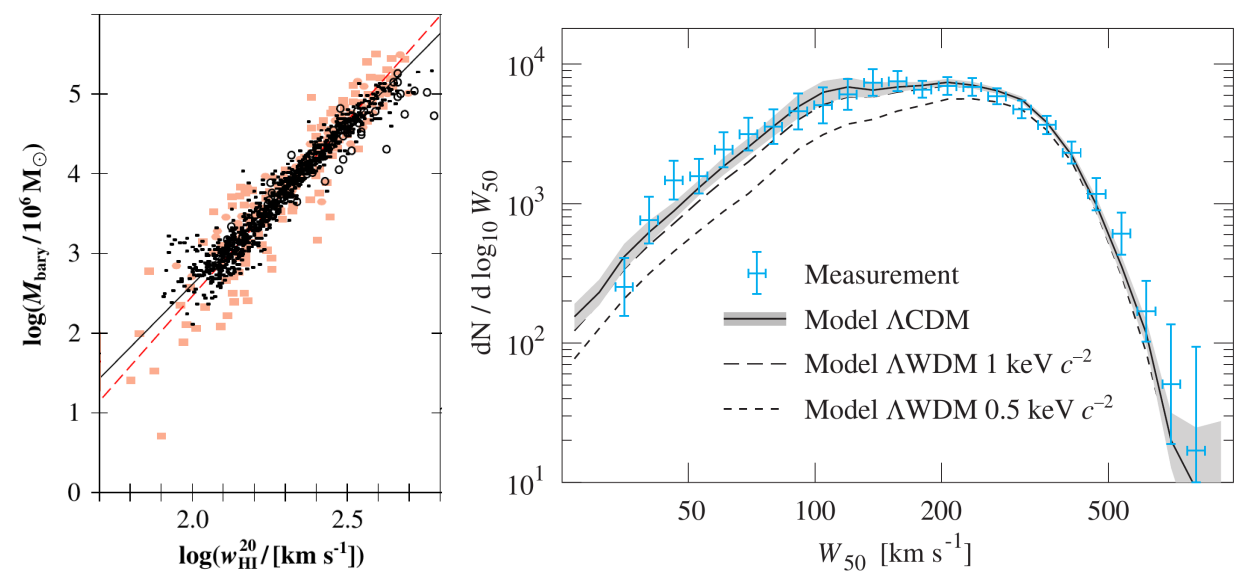

Figure 8: The left panel shows the baryonic mass as a function of HI velocity width measured at 0.2 of peak flux, $w_{20}$. Simulated spirals and elliptical galaxies from Obreschkow et al. (2009) are shown as filled and empty circles, respectively, while observations from McGaugh et al. (2000) are shown as solid squares. The right panel shows the distribution of HI velocity widths measured at 0.5 of peak flux, $w_{50}$ for the simulation of Obreschkow et al. (2009). Observations from Zwaan et al. (2010) are shown as symbols.

emission lines using predicted galaxy properties and to replicate observations using model data.

The ability to study evolution in the internal kinematics of galaxies offer new and powerful insights into a property of galaxies that has traditionally been difficult to study but which plays a crucial role in shaping not only a galaxy's morphology but also the distribution of gas, the impact of feedback, the growth of its supermassive black hole, etc... namely angular momentum. This will be an area in which the SKA can have a transformational impact.

\section{Future Developments}

We now survey anticipated developments in the models, focussing on three key areas. First, the distribution of gas in the circum- and inter-galactic media (CGM and IGM respectively); second, feedback within galaxies; and third next generation synthetic surveys.

Distribution of Gas in the CGM and IGM. Large HI $21 \mathrm{~cm}$ emission line surveys of local galaxies (Zwaan et al., 2005) and absorption-line measurements in the spectra of quasi-stellar objects (Noterdaeme et al., 2012) have allowed accurate measurement of the evolution of the global density of atomic hydrogen over the redshift range $0 \leq z \lesssim 4$, and these suggest little evolution of the global density of atomic hydrogen, $\Omega_{\mathrm{HI}}$, with time. The latest semi-analytics models account for $\mathrm{HI}$ in galaxies and can account for evolution of $\Omega_{\mathrm{HI}}$ over the range $0 \leq z \lesssim 2.5$, but predict lower values than observed at higher redshifts by a factor of $\sim 2$, suggesting a large reservoir of neutral hydrogen outside of galaxies (Lagos et al., 2011a). This neutral gas does not need to be outside of the galaxy's halo, but instead it could be in the CGM. Indeed, observations indicate that it is within $\approx 20-40 \mathrm{kpc}$ of the galaxy (Krogager et al., 2012).

The interpretation of the results in Lagos et al. (2012) are consistent with the results of recent hydro-dynamical simulations (van de Voort et al., 2012; Davé et al., 2013; Rahmati \& Schaye, 2014). The challenge for semi-analytical models is to account for gas in the CGM and IGM in a 
self-consistent way. One approach will be to couple semi-analytical models to a hydrodynamical description of the IGM. Such a technique has been recently applied by Moster et al. (2014) to idealised galaxy mergers. On larger scales, work is already underway (by some of the authors) to characterise the sensitivity of the IGM to the choice of cooling and feedback prescriptions adopted in hydrodynamical simulations, to develop a simple predictive model for IGM properties based on the underlying dark matter density field. A complementary approach is to use state-of-theart gas dynamical simulations to study the distribution of neutral gas in haloes combined with semi-analytical calculations of the neutral gas distribution at cosmological reionization (Kim et al., 2013a). Both techniques will lead to an ultimate answer to what is the predicted density of HI all the way from $z=0$ to $z=12$ including all possible sources of neutral gas in an ab-initio galaxy formation simulation. A natural by-product of this work will be predictions for $\mathrm{HI}$ absorption.

Impact of Stellar Feedback. Feedback, the process by which stars and accreting compact objects interact with and modify their environment, is what makes galaxy formation modelling particularly challenging. In particular, feedback from supernovae $(\mathrm{SNe})$ represents a long standing problem. Traditionally it has been parametrized to reproduce the faint-end of the luminosity function (Guo et al., 2011), but ignored physical properties of the galaxy such as the density of the ISM or the amount of energy released. Recent models (Creasey et al., 2013; Hopkins et al., 2012; Lagos et al., 2013) now incorporate a treatment of SNe-driven outflows that account for the physical properties of the galaxy, and have, for example, revealed the importance of gas surface density in regulating mass entrained in the outflows, which was ignored in previous models.

This improved description gives predictions of mass entrainment and outflow velocities in good agreement with observations. However, the development of a galaxy-wide outflow does not necessarily imply long-term disruption of the galaxy's gas reservoir, and improvements are necessary to calculate the evolution of such outflows - when are they confined by the galaxy's halo, and when do they escape? These question also relate to the condition for gas expelled from the galaxy to be reincorporated into it. Whether or not gas that is expelled from the disc returns to the galaxy or is lost to the gaseous halo is one of the key unknowns in galaxy formation, which lead to very different star formation histories of galaxies. For example, Henriques et al. (2013) showed that a different parametric form for reincorportation will help the build-up of the stellar mass predicted by models to get closer that what is inferred from observations. Resolving this problem will require not only extensions of the models but careful comparison with gas-dynamical simulations of galaxies in both a controlled non-cosmological setting and in cosmological simulations.

Next Generation Synthetic Surveys. The importance of the models in the design and astrophysical interpretation of future surveys means that the creation of synthetic surveys is central to future developments of the models. We focus on two aspects. First, radio continuum emission. The SKA is predicted to detect hot, diffuse gas in the cosmic web, undetectable with current instruments, through its emission in the radio continuum. At the same time, these surveys will detect radio continuum emission from galaxies, driven by star formation and AGN. Some of the framework to calculate radio continuum emission is already in place, e.g. the work of Fanidakis et al. (2011) on the mass and spin growth of black hole engines of AGN. However, further development is required to account for emission from star forming regions and the cosmic web, involving e.g. hybrid 
hydrodynamical and semi-analytical modelling. Second, molecular line emission. The SKA will survey molecular emission from the high-redshift Universe (Geach \& Papadopoulos, 2012) using the novel technique of intensity mapping (Carilli, 2011). This gives two-dimensional spatial information about the emission line at a given redshift; imaging is obtained by aggregating emission from thousands of galaxies on very large scales to produce the summed signal of galaxies that are not individually detected. Future models must account for e.g. thermalisation of the ISM of galaxies by the cosmic microwave background and e.g. the full spectral line energy distribution of the most used molecular emission line, carbon monoxide (CO).

\section{Testing Dark Matter Models with the SKA}
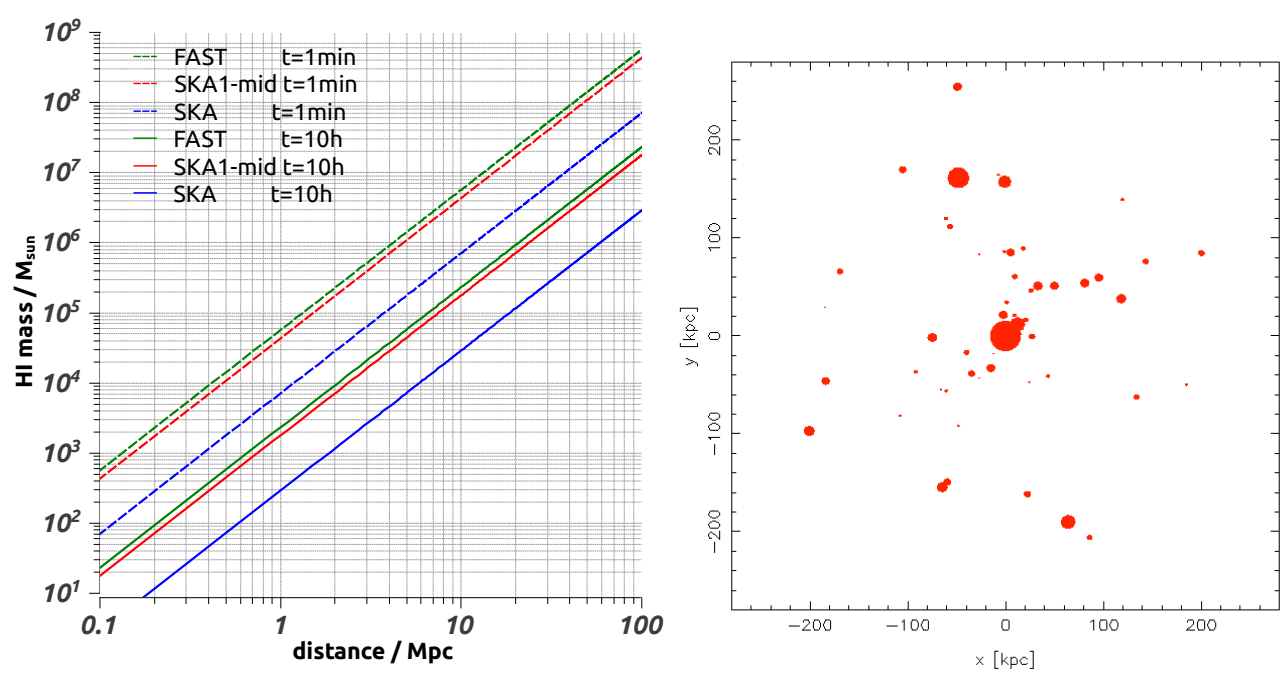

Figure 9: Left Panel: HI detection limits of FAST (green), SKA1-MID (red), and SKA2 (blue). Dashed and solid lines show $1 \mathrm{~min}$ and $10 \mathrm{~h}$ integrations. Right Panel: A preliminary result of the HI distributions of a Milky Way-like galaxy from the Aquarius simulation. The HI mass limit is $10^{4} \mathrm{M}_{\odot}$.

The CDM model for cosmological structure formation is well established. However, accumulating evidence during the past 15 years has revealed that its predictions are at odds with observations on galactic and sub-galactic scales. Numerical simulations of the CDM model predict abundances of substructure halos (subhalos) that are an order of magnitude larger than the numbers of satellite galaxies observed around nearby galaxies. This has led to modifications of CDM to be proposed, ranging from coupling between CDM and radiation (Bœhm et al., 2014) to self-interacting dark matter (Spergel \& Steinhardt, 2000; Qin \& Wu, 2001) to Warm Dark Matter (WDM; cf. Bode et al., 2001), all of which suppress the abundance of subhalos. For example, Lovell et al. (2014) have shown that the subhalo abundance in a WDM galaxy-mass halo at $\mathrm{M}_{\text {sub }}>10^{7} \mathrm{M}_{\odot}$ is approximately a factor of 10 smaller than in the corresponding CDM halo, and Kennedy et al. (2014) have shown that the magnitude of the effect depends strongly on WDM particle mass.

HI observations may reveal optically invisible satellite galaxies around nearby galaxies. For example, the Arecibo ALFALFA HI survey of the local Universe (Haynes et al., 2011) has found more than 15,000 extragalactic HI sources within $\sim 250 \mathrm{Mpc}$, with the lowest HI mass of a few 
$10^{6} \mathrm{M}_{\odot}$. SKA1-MID will have higher sensitivity and a much larger field of view (FoV, $0.7 \mathrm{deg}$ $\times 0.7 \mathrm{deg}$ ) than Arecibo; SKA2 will 10 times higher sensitivity and 20 times larger FoV. The left panel of Fig. 9 shows the HI detection limits for the planned 500-m FAST (Nan et al., 2011), SKA1-MID, and SKA2, at $1 \mathrm{~min}$ and 10 hours integrations, with velocity linewidth of $200 \mathrm{~km} / \mathrm{s}$ and signal-to-noise of 6. SKA1-MID (SKA2) will be able to detect HI masses of a few $10^{6} \mathrm{M}_{\odot}$ $\left(10^{5} \mathrm{M}_{\odot}\right)$ respectively at a distance of $50 \mathrm{Mpc}$ and with a 10 -hour integration; this corresponds to host halo masses of order $\sim 10^{7}-10^{8} \mathrm{M}_{\odot}$, assuming that these halos contain a few percent of HI mass. This implies that the SKA can potentially discriminate between the CDM and WDM models by detecting the difference in predicted subhalo abundance.

The right panel of Fig. 9 shows the predicted distribution of HI gas in satellites in a Milky Way-type halo, taken from the Aquarius Simulation (Springel et al., 2008) coupled to the semianalytical model of Fu et al. (2010), with an HI cutoff mass of $10^{4} \mathrm{M}_{\odot}$; the influence of reionization is accounted for, and dot size is proportional to HI mass. This indicates that the optically invisible satellite haloes of nearby galaxies may be visible by the SKA, if they contain enough HI gas. By surveying $\sim 100$ nearby galaxies within a few tens Mpc, and comparing with the results of numerical simulations such as the Aquarius Simulation, the SKA may be able to provide independent limits on dark matter particle candidates and, in doing so, guide experimental astro-particle physicists in their efforts to detect the dark matter particle directly in the lab.

\section{Summary}

Neutral hydrogen plays a fundamental role in galaxy formation, as the raw material from which stars form and galaxies are built, yet much of what we know about the $\mathrm{HI}$ and $\mathrm{H}_{2}$ content of galaxies derives from the local Universe. The SKA will change this dramatically, revolutionising our understanding of neutral hydrogen in galaxies over cosmic time and its role in galaxy formation and evolution. Recognition of this has led to rapid developments in theoretical galaxy formation models over the last $\sim 5$ years, which we have reviewed. Key has been a radical rewriting of the way star formation is modelled (Lagos et al., 2011b), linking explicitly a galaxy's star formation rate to its $\mathrm{H}_{2}$ abundance, in agreement with both high resolution observations of star-forming regions in galaxies (e.g. Bigiel et al. 2008) and the results of detailed numerical simulations (e.g. Glover \& Clark 2012b).

This has resulted in pleasing consistency between model predictions (e.g. Lagos et al. 2011a; Kim et al. 2011) and observations of HI in galaxies in the local Universe (e.g. Zwaan et al. 2005; Meyer et al. 2007; Martin et al. 2010), as well as providing insights into how gas is distributed in the high redshift Universe (e.g. Lagos et al. 2012). At the same time the models have indicated the kind of observations that are necessary to better understand the physical processes that drive galaxy formation, most notably feedback (Kim et al., 2013b), and to constrain dark matter particle physics by placing limits on the abundance of low-mass dark matter halos containing cold gas.

The models are still evolving, however, and we have highlighted key areas where further developments, including the development of hybrid schemes in which semi-analytics and gas dynamical simulations are coupled to better capture the distribution of gas outside of galaxies and how this gas accretes onto galaxies; the modelling of feedback from supernovae; and more realistic synthetic galaxy surveys. These are topics where the authors will be focussing their collective efforts in the coming years. 


\section{References}

Angulo, R. E., Springel, V., White, S. D. M., et al. 2012, MNRAS, 426, 2046

Barnes, D. G., Staveley-Smith, L., de Blok, W. J. G., et al. 2001, MNRAS, 322, 486

Baugh, C. M. 2006, Reports on Progress in Physics, 69, 3101

Baugh, C. M., Lacey, C. G., Frenk, C. S., et al. 2004, New Astron. Rev., 48, 1239

-. 2005, MNRAS, 356, 1191

Bekki, K. 2014, MNRAS, 438, 444

Benson, A. J., Bower, R. G., Frenk, C. S., et al. 2003, ApJ, 599, 38

Benson, A. J., Lacey, C. G., Baugh, C. M., Cole, S., \& Frenk, C. S. 2002, MNRAS, 333, 156

Berta, S., Lutz, D., Nordon, R., et al. 2013, ArXiv:1304.7771, arXiv:1304.7771

Bigiel, F., Leroy, A., Walter, F., et al. 2008, AJ, 136, 2846

Blitz, L., \& Rosolowsky, E. 2006, ApJ, 650, 933

Bode, P., Ostriker, J. P., \& Turok, N. 2001, ApJ, 556, 93

Bohm, C., Schewtschenko, J. A., Wilkinson, R. J., Baugh, C. M., \& Pascoli, S. 2014, MNRAS, 445, L31

Book, L. G., \& Benson, A. J. 2010, ApJ, 716, 810

Bower, R. G., Benson, A. J., Malbon, R., et al. 2006, MNRAS, 370, 645

Boylan-Kolchin, M., Springel, V., White, S. D. M., Jenkins, A., \& Lemson, G. 2009, MNRAS, 398,1150

Carilli, C. L. 2011, ApJ L, 730, L30

Cole, S., Lacey, C. G., Baugh, C. M., \& Frenk, C. S. 2000, MNRAS, 319, 168

Cook, M., Evoli, C., Barausse, E., Granato, G. L., \& Lapi, A. 2010, MNRAS, 402, 941

Creasey, P., Theuns, T., \& Bower, R. G. 2013, MNRAS, 429, 1922

Croton, D. J., Springel, V., White, S. D. M., et al. 2006, MNRAS, 365, 11

Davé, R., Katz, N., Oppenheimer, B. D., Kollmeier, J. A., \& Weinberg, D. H. 2013, ArXiv:1302.3631, arXiv:1302.3631

Davé, R., Oppenheimer, B. D., \& Finlator, K. 2011, MNRAS, 415, 11

de Blok, E., Booth, R., \& Frank, B. 2009, in Panoramic Radio Astronomy: Wide-field 1-2 GHz

Research on Galaxy Evolution

De Lucia, G., \& Blaizot, J. 2007, MNRAS, 375, 2

Delhaize, J., Meyer, M., Staveley-Smith, L., \& Boyle, B. 2013, ArXiv:1305.1968, arXiv:1305.1968

Dobbs, C. L., Burkert, A., \& Pringle, J. E. 2011, MNRAS, 417, 1318

Dutton, A. A., van den Bosch, F. C., \& Dekel, A. 2010, MNRAS, 405, 1690

Fanidakis, N., Baugh, C. M., Benson, A. J., et al. 2011, MNRAS, 410, 53

Feldmann, R., Gnedin, N. Y., \& Kravtsov, A. V. 2011, ApJ, 732, 115

Font, A. S., Bower, R. G., McCarthy, I. G., et al. 2008, MNRAS, 389, 1619

Fu, J., Guo, Q., Kauffmann, G., \& Krumholz, M. R. 2010, MNRAS, 409, 515

Geach, J. E., \& Papadopoulos, P. P. 2012, ApJ, 757, 156

Geach, J. E., Smail, I., Moran, S. M., et al. 2011, ApJ L, 730, L19+

Giovanelli, R., Haynes, M. P., Kent, B. R., et al. 2005, AJ, 130, 2598

Glover, S. C. O., \& Clark, P. C. 2012a, MNRAS, 421, 116

-. 2012b, MNRAS, 421, 9 
Gnedin, N. Y., \& Kravtsov, A. V. 2011, ApJ, 728, 88

Guo, Q., White, S., Boylan-Kolchin, M., et al. 2011, MNRAS, 413, 101

Haynes, M. P., Giovanelli, R., Martin, A. M., et al. 2011, AJ, 142, 170

Henriques, B. M. B., White, S. D. M., Thomas, P. A., et al. 2013, MNRAS, 431, 3373

Hopkins, P. F., Quataert, E., \& Murray, N. 2012, MNRAS, 421, 3522

Johnston, S., Taylor, R., Bailes, M., et al. 2008, Experimental Astronomy, 22, 151

Kennedy, R., Frenk, C., Cole, S., \& Benson, A. 2014, MNRAS, 442, 2487

Keres, D., Yun, M. S., \& Young, J. S. 2003, ApJ, 582, 659

Kim, H.-S., Baugh, C. M., Benson, A. J., et al. 2011, MNRAS, 414, 2367

Kim, H.-S., Power, C., Baugh, C. M., et al. 2013a, MNRAS, 428, 3366

Kim, H.-S., Wyithe, J. S. B., Raskutti, S., Lacey, C. G., \& Helly, J. C. 2013b, MNRAS, 428, 2467

Krogager, J.-K., Fynbo, J. P. U., Møller, P., et al. 2012, MNRAS, 424, L1

Krumholz, M. R. 2013, MNRAS, 436, 2747

Krumholz, M. R., McKee, C. F., \& Tumlinson, J. 2009, ApJ, 699, 850

Lagos, C. D. P., Baugh, C. M., Lacey, C. G., et al. 2011a, MNRAS, 418, 1649

Lagos, C. D. P., Baugh, C. M., Zwaan, M. A., et al. 2014, MNRAS, 440, 920

Lagos, C. d. P., Bayet, E., Baugh, C. M., et al. 2012, MNRAS, 426, 2142

Lagos, C. d. P., Lacey, C. G., \& Baugh, C. M. 2013, MNRAS, 436, 1787

Lagos, C. D. P., Lacey, C. G., Baugh, C. M., Bower, R. G., \& Benson, A. J. 2011b, MNRAS, 416, 1566

Lah, P., Chengalur, J. N., Briggs, F. H., et al. 2007, MNRAS, 376, 1357

Lovell, M. R., Frenk, C. S., Eke, V. R., et al. 2014, MNRAS, 439, 300

Martin, A. M., Papastergis, E., Giovanelli, R., et al. 2010, ApJ, 723, 1359

Martin, C. L., Shapley, A. E., Coil, A. L., et al. 2012, ApJ, 760, 127

McGaugh, S. S., Schombert, J. M., Bothun, G. D., \& de Blok, W. J. G. 2000, ApJ L, 533, L99

Meyer, M. J., Zwaan, M. A., Webster, R. L., Brown, M. J. I., \& Staveley-Smith, L. 2007, ApJ, 654, 702

Moster, B. P., Macciò, A. V., \& Somerville, R. S. 2014, MNRAS, 437, 1027

Nan, R., Li, D., Jin, C., et al. 2011, International Journal of Modern Physics D, 20, 989

Noterdaeme, P., Petitjean, P., Carithers, W. C., et al. 2012, A\&A, 547, L1

Obreschkow, D., Croton, D., De Lucia, G., Khochfar, S., \& Rawlings, S. 2009, ApJ, 698, 1467

Obreschkow, D., Ma, X., Meyer, M., et al. 2013, ApJ, 766, 137

Obreschkow, D., \& Rawlings, S. 2009, MNRAS, 394, 1857

Power, C., Baugh, C. M., \& Lacey, C. G. 2010, MNRAS, 406, 43

Putman, M. E., Peek, J. E. G., \& Joung, M. R. 2012, ARA\&A, 50, 491

Qin, B., \& Wu, X.-P. 2001, Physical Review Letters, 87, 061301

Quilis, V., Moore, B., \& Bower, R. 2000, Science, 288, 1617

Rahmati, A., \& Schaye, J. 2014, MNRAS, 438, 529

Rawlings, S., Abdalla, F. B., Bridle, S. L., et al. 2004, New Astronomy Reviews, 48, 1013

Robertson, B. E., \& Kravtsov, A. V. 2008, ApJ, 680, 1083

Schaye, J. 2004, ApJ, 609, 667

Schaye, J., Crain, R. A., Bower, R. G., et al. 2014, ArXiv e-prints, arXiv:1407.7040

Sobral, D., Smail, I., Best, P. N., et al. 2013, MNRAS, 428, 1128 
Speagle, J. S., Steinhardt, C. L., Capak, P. L., \& Silverman, J. D. 2014, ApJS, 214, 15

Spergel, D. N., \& Steinhardt, P. J. 2000, Physical Review Letters, 84, 3760

Springel, V., White, S. D. M., Jenkins, A., et al. 2005, Nat, 435, 629

Springel, V., Wang, J., Vogelsberger, M., et al. 2008, MNRAS, 391, 1685

van Daalen, M. P., Schaye, J., McCarthy, I. G., Booth, C. M., \& Vecchia, C. D. 2014, MNRAS, 440, 2997

van de Voort, F., Schaye, J., Altay, G., \& Theuns, T. 2012, MNRAS, 421, 2809

Vogelsberger, M., Genel, S., Springel, V., et al. 2014, Nat, 509, 177

Wong, T., \& Blitz, L. 2002, ApJ, 569, 157

Wyithe, J. S. B., \& Brown, M. J. I. 2010, MNRAS, 404, 876

Zwaan, M. A., Meyer, M. J., \& Staveley-Smith, L. 2010, MNRAS, 403, 1969

Zwaan, M. A., Meyer, M. J., Staveley-Smith, L., \& Webster, R. L. 2005, MNRAS, 359, L30

Zwaan, M. A., Staveley-Smith, L., Koribalski, B. S., et al. 2003, AJ, 125, 2842 